\title{
SIMULAÇÃO NUMÉRICA DA ADSORÇÃO DE METANO E NITROGENIO EM LEITO FIXO CONTENDO SILICALITA
}

\author{
H.R. Sant Anna ${ }^{1}$, A.G. Barreto Jr. ${ }^{1}$, F.W. Tavares ${ }^{1,2}$, C.R.A. Abreu ${ }^{1}$ e J.F. do Nascimento ${ }^{3}$ \\ ${ }^{1}$ Universidade Federal do Rio de Janeiro, Escola de Química \\ ${ }^{2}$ Universidade Federal do Rio de Janeiro, Instituto Alberto Luiz Coimbra de Pós-graduação e \\ Pesquisa em Engenharia, Programa de Engenharia Química \\ ${ }^{3}$ Petrobras, CENPES / PDEP / TPP \\ E-mail para contato: hermes@eq.ufrj.br
}

\begin{abstract}
RESUMO - O presente trabalho trata da simulação numérica da dinâmica de adsorção de uma mistura binária $\mathrm{CH}_{4} / \mathrm{N}_{2}$ em um leito fixo de adsorção empacotado com silicalita. $\mathrm{O}$ modelo empregado é um sistema de equações diferenciais parciais, formado por balanços de massa e energia bem como equações algébricas, que traduzem equações constitutivas selecionadas. O modelo é resolvido empregando-se o método das linhas. Curvas de ruptura experimentais disponíveis na literatura foram usadas para a verificação da capacidade de previsão, considerando-se a descrição de perfis obtidos com substâncias puras e com misturas binárias. A modelagem serve para nortear o dimensionamento de uma unidade de adsorção em escala de bancada, oferecendo subsídios para a especificação do sistema de troca térmica e posicionamento de instrumentos analíticos para a medição de temperaturas e composições.
\end{abstract}

\section{INTRODUÇÃO}

Rejeitado como subproduto na produção de petróleo até metade do século XX, o gás natural já responde por $23 \%$ do consumo mundial de energia. Sendo uma fonte de energia eficiente e ambientalmente mais correta, este é o combustível convencional de queima mais limpa. No entanto, para seu consumo como produto final, o gás extraído da cabeça de poço necessita da remoção de diversos contaminantes, tais como $\mathrm{CO}_{2}, \mathrm{H}_{2} \mathrm{O}$ e $\mathrm{N}_{2}$. Por possuir propriedades físico-químicas tão próximas do metano, principal constituinte do gás natural, a remoção deste último é um dos maiores desafios enfrentados pela indústria nos dias de hoje. Por ser um gás inerte na queima do combustível em questão, o excesso de nitrogênio pode causar uma drástica redução do poder calorífico do gás natural, além de elevar os custos de compressão e de transporte por gasodutos. Cerca de $16 \%$ das reservas dos EUA apresentam nitrogênio como contaminante, sendo muitas delas comercialmente inviáveis (Kuo et al,. 2012 e Lokhandwala et al., 2010). Já no Brasil, poços contaminados com este inerte podem ser encontrados nas regiões norte e nordeste (Madeira, 2008). A tecnologia mais madura para a remoção de $\mathrm{N}_{2}$ é a destilação criogênica, única opção comercialmente viável para produção de gás com tal contaminante em largas escalas (> 700 $\mathrm{MNm}^{3} /$ dia) (Kidnay \& Parrish, 2006). Através de expansões Joule-Thomson, este tipo de unidade de remoção de nitrogênio (URN) promove a 


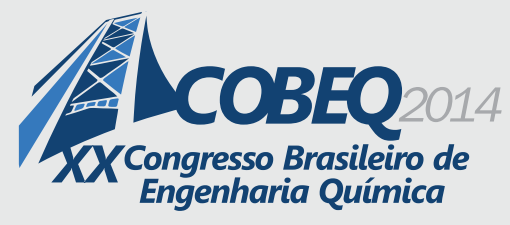

19 a 22 de outubro de 2014

Florianópolis/SC

separação por equilíbrio líquido-vapor a temperaturas tão baixas quanto $-150^{\circ} \mathrm{C}$, gerando um produto com baixo teor de $\mathrm{N}_{2}$. No entanto, a depender do processo escolhido e do teor dos demais contaminantes no gás natural, a corrente de nitrogênio que sai da unidade pode conter quantidades significativas de $\mathrm{CH}_{4}$. Por outra perspectiva, é possível extrair o $\mathrm{N}_{2}$ do gás natural de reservatórios de baixa produção utilizando permeação por membranas ou PSA (pressure swing adsorption). No tocante ao PSA, apesar do gás natural apresentar, em geral, uma fração molar de $\mathrm{CH}_{4}$ maior que a de $\mathrm{N}_{2}$, adsorventes como carvão ativado e peneira molecular de carbono apresentam seletividade ao metano e não ao nitrogênio. Por outro lado, adsorventes da família dos titanosilicatos, como ETS4, e das clinoptilolitas podem apresentar seletividades cinéticas ao $\mathrm{N}_{2}$ em detrimento do metano. Porém, o uso destes adsorventes em unidades industriais para o processamento de vazões altas ainda se apresenta inviável sob o ponto de vista econômico.

Neste contexto, as características dos processos citados anteriormente abrem margem para a proposição de um processo híbrido de remoção de nitrogênio. Devido às seletividades termodinâmicas dos sólidos conhecidos favorecerem a adsorção de metano, eles podem ser utilizados para a remoção deste componente a partir das correntes de rejeito das unidades de destilação criogênica. Deste modo, é possível a utilização de colunas de destilação menores, operando em temperaturas menos extremas e que emitem menos poluentes para a atmosfera. Neste sentido, o presente trabalho se propõem a analisar o comportamento dinâmico de uma unidade de adsorção em leito fixo a partir da implementação numérica da modelagem de leito fixo apresentado na literatura (Ruthven, 1984). O comportamento do modelo matemático é verificado em relação à inclusão do balanço de massa global e à inclusão dos efeitos de competição de $\mathrm{CH}_{4}$ e $\mathrm{N}_{2}$ pelo adsorvente. A inclusão de balanço de energia permite analisar os possíveis efeitos térmicos envolvidos no processo, que podem ser bastante relevantes para a integração com o processo criogênico. Simulações foram desenvolvidas para avaliação da magnitude da temperatura ao longo da coordenada axial do leito para uma unidade em escala de laboratório. Os resultados obtidos estão sendo usados para nortear a especificação de equipamentos analíticos para a montagem de um sistema experimental.

\section{SIMULAÇÃO NUMÉRICA}

A análise da dinâmica do leito adsortivo, empacotado com silicalita, foi realizada a partir da solução numérica de balanços de massa, energia e demais equações constitutivas, que descrevem o escoamento, o comportamento de equilíbrio e cinética da adsorção. A modelagem matemática foi realizada baseada nas seguintes premissas: escoamento em plug flow com dispersão axial; velocidade variável para o fluido; coluna não isotérmica (equilíbrio térmico local entre o sólido e o gás é assumido); cinética representada pelo modelo LDF (Linear Driving Force); fase gasosa apresenta comportamento de gás ideal; gradientes de concentração e temperatura radiais são desprezíveis; pressão uniforme e constante ao longo da coluna. Neste contexto, a equação do balanço de massa no leito por componente é dada por (Ruthven, 1984):

$$
-D_{a x} \frac{\partial^{2} c_{i}}{\partial z^{2}}+\frac{\partial\left(v c_{i}\right)}{\partial z}+\frac{\partial c_{i}}{\partial t}+\frac{1-\varepsilon}{\varepsilon} \frac{\partial q_{i}}{\partial t}=0
$$

em que $D_{a x}$ é o coeficiente de dispersão axial, $c_{i}$ a concentração dos constituintes da mistura, $z$ a 
coordenada axial, $v$ a velocidade superficial, $q_{i}$ a concentração adsorvida e $\varepsilon$ a fração de vazios do leito. $\mathrm{O}$ balanço material global tem a forma

$$
\frac{\partial(v C)}{\partial z}+\frac{\partial C}{\partial t}+\frac{1-\varepsilon}{\varepsilon} \sum_{i=1}^{n} \frac{\partial q_{i}}{\partial t}=0
$$

e $C$ é a concentração total da fase gasosa, que é dada por

$$
C=\frac{P}{R T}
$$

Os mecanismos de transporte de massa no sólido adsorvente são representados pela abordagem LDF, como descrito pela Equação (4).

$$
\frac{\partial q_{i}}{\partial t}=k_{i}\left(q_{i}^{*}-q_{i}\right)
$$

em que $k_{i}$ é o coeficiente de transferência de massa agrupado, $q_{i}{ }^{*}$ a concentração adsorvida em equilíbrio com a fase fluida. O balanço de energia na coluna é descrito pela Equação (5) (Ruthven, 1984).

$$
-K_{a x} \frac{\partial^{2} T}{\partial z^{2}}+C_{g} \frac{\partial(v T)}{\partial z}+\left(C_{g}+\frac{1-\varepsilon}{\epsilon} C_{s}\right) \frac{\partial T}{\partial t}-\sum_{i}\left(-\Delta H_{i}\right) \frac{1-\varepsilon}{\varepsilon} \frac{\partial q_{i}}{\partial t}+\frac{2 h}{\varepsilon r_{i n}}\left(T-T_{W}\right)=0
$$

Nesta, $K_{a x}$ é o coeficiente de dispersão axial térmico, $T$ a temperatura do sólido e do fluido, $C_{g}$ o calor específico do gás, $C_{s}$ o calor específico do adsorvente, $\Delta H_{i}$ a entalpia de adsorção do componente $\mathrm{i}, h$ o coeficiente de transferência de calor entre o gás e a parede, $r_{i n}$ o raio interno da coluna e $T_{w}$ a temperatura da parede da coluna. O equilíbrio de adsorção da mistura pode ser modelado por duas formas diferentes da isoterma de Langmuir, uma desconsiderando o efeito da competição das moléculas por sítios ativos na interface do sólido e outra considerando tal efeito. A isoterma de Langmuir sem o efeito de competição é dada por:

$$
\frac{q_{i}}{q_{i, s}}=\frac{b_{i} p_{i}}{1+b_{i} p_{i}}
$$

onde $q_{i}$ é a concentração do componente i na fase adsorvida, $q_{i s}$ é a concentração do componente i em condições de saturação do leito e $b_{i}$ é a constante de afinidade e $p_{i}$ a pressão parcial do componente i na fase gasosa. Adicionando o efeito da competição das espécies pelo sítio ativo, o modelo se torna:

$$
\frac{q_{i}}{q_{i, s}}=\frac{b_{i} p_{i}}{1+\sum_{j} b_{j} p_{j}}
$$

A constante de Henry $b_{i}$ é dependente da temperatura, sendo sua fórmula baseada na integração da equação de vant Hoff, gerando (Ruthven, 1984):

$$
b_{i}=b_{i, 0} * e^{\left(-\frac{\Delta H_{i}}{R} *\left(\frac{1}{T}-\frac{1}{T_{0}}\right)\right)}
$$




\section{9 a 22 de outubro de 2014 \\ Florianópolis/SC}

onde $b_{i, 0}$ é a constante de Henry ("afinidade") na temperatura de referência, $-\Delta H_{i}$ a diferença de entalpia entre a fase adsorvida e gasosa, $T$ a temperatura do sistema e $T_{0}$ a temperatura de referência.

O leito foi dividido em elementos finitos, os quais foram particionados em dois sub-elementos. Deste modo, torna-se possível a utilização de aproximações parabólicas para os perfis de temperatura e concentração por componente, viabilizando a discretização espacial de suas primeiras e segundas derivadas. Para a discretização das demais derivadas espaciais de primeira ordem, foi utilizado o método das diferenças finitas. Nas coordenadas espaciais, as aproximações anteriormente mencionadas geraram um sistema de equações algébricas tri-diagonal, sendo utilizado o método de Thomas para sua resolução. Já na dimensão temporal, o sistema de equações algébrico-diferenciais foi integrado utilizando a rotina DDASSL (Petzold et al., 1983). O sistema de equações do modelo foi implementado na linguagem de programação FORTRAN 90. O adsorvente simulado no leito fixo foram pellets de silicalita analisados por Delgado et al. (2006). A Tabela 1 apresenta as constantes da isoterma de equilíbrio ajustadas experimentalmente pelo autor, bem como as constantes da abordagem LDF (Farooq e Ruthven, 1990).

Tabela 1 - Valores dos parâmetros do sistema adsortivo

\begin{tabular}{llr}
\hline & $\mathrm{N}_{2}$ & $\mathrm{CH}_{4}$ \\
Constante de afinidade (298 K), b $\left(10^{-7} \mathrm{~mol} \mathrm{~kg}^{-1} \mathrm{~Pa}^{-1}\right)$ & 9,13 & 30,8 \\
Capacidade de adsorção máxima, qs $\left(\mathrm{mol} \mathrm{kg}^{-1}\right)$ & 1,73 & 1,73 \\
Entalpia de adsorção, $-\Delta H_{i}\left(\mathrm{~kJ} \mathrm{~mol}^{-1}\right)$ & 14,9 & 18 \\
Constante do modelo LDF, $k_{i}\left(10^{3} \mathrm{~s}^{-1}\right)$ & 1,67 & 1,5 \\
\hline
\end{tabular}

\section{RESULTADOS E DISCUSSÃO}

Dois leitos foram simulados, um com dimensões correspondentes ao texto de referência para verificação (Delgado et al., 2006) e outro com dimensões para análise da modelagem matemática, conforme apresentado na Tabela 2. Uma vez que foi utilizada uma isoterma de adsorção diferente da apresentada pelo autor de base, a modelagem apresentada no Capítulo 2 foi verificada pela comparação da curva de ruptura prevista pelo modelo descrito contra dados experimentais do trabalho citado. Na Figura 1, são comparados pontos experimentais obtidos pelo autor contra curvas as curvas calculadas, relativas às frações molares de $\mathrm{CH}_{4}$ na saída do leito em questão. Pode-se observar boa concordância entre os dados experimentais e a curva teórica.

Em uma análise posterior, avaliou-se a influência da variação da velocidade do fluido na dinâmica do leito fixo. A Figura 2 (a) apresenta as curvas de ruptura representando o perfil de concentração da fase gasosa na saída do leito a $25^{\circ} \mathrm{C}$ e 1,0 bar, alimentado por uma corrente contendo $20 \%$ de $\mathrm{CH}_{4}$. Nela, é a presentado o perfil de concentração de $\mathrm{CH}_{4}$ e $\mathrm{N}_{2}$ na saída do leito (C, $\left.\left[\mathrm{mol} / \mathrm{m}^{3}\right]\right)$, divididas por suas respectivas concentrações na corrente de alimentação $\left(\mathrm{C}_{0},\left[\mathrm{~mol} / \mathrm{m}^{3}\right]\right)$. É possível observar uma clara diferença entre as curvas do $\mathrm{N}_{2}$ que, à velocidade constante, apresentou uma forma sigmoidal simples. Entretanto, considerando a variação da velocidade, ocorreu uma sobreelevação a concentrações superiores à de entrada. Tal forma demonstra o efeito de deslocamento. Sendo o $\mathrm{N}_{2}$ o componente menos retido (menor afinidade pelo adsorvente), sua frente de massa se 
movimenta mais rapidamente, no entanto tais moléculas adsorvidas são deslocadas da superfície do sólido para o fluido pela adsorção de metano com o avanço de sua frente de massa, elevando a concentração do $\mathrm{N}_{2}$ na saída.

Tabela 2 - Especificações das colunas simuladas

Diâmetro, $D(\mathrm{~m})$

Comprimento, $L(\mathrm{~m})$

Porosidade total, $\varepsilon_{t}$

Vazão, $Q\left(10^{-7} \mathrm{~m}^{3} \mathrm{~s}^{-1}\right)$

Difusividade axial, $\operatorname{Dax}\left(10^{-5} \mathrm{~m}^{2} \mathrm{~s}^{-1}\right)$

Difusividade térmica, $K a x\left(10^{-4} \mathrm{~kJ} \mathrm{~m}^{2} \mathrm{~mol}^{-1} \mathrm{~K}^{-1} \mathrm{~s}^{-1}\right)$

Coef. Radial de transf, de calor, $h\left(10^{3} \mathrm{~kW} \mathrm{~m}^{-1} \mathrm{~K}^{-1}\right)$

$\begin{array}{rrl}\text { Verificação } & \text { Análise } & \\ 0,016 & 0,01 & \\ 0,163 & 0,1 & \\ 0,52 & 0,52 & \\ - & 1,23 & \\ 5,82 & 2,55 & \text { (Farooq \& Ruthven 1990) } \\ 2,71 & 2,71 & \text { (Farooq \& Ruthven 1990) } \\ 1,17 & 1,09 & \text { (De Wasch \& Froment 1972) }\end{array}$

Tabela 3 - Casos para verificação do modelo

\begin{tabular}{lllll} 
Caso & $P_{0}($ bar $)$ & $y_{a} \mathrm{CH}_{4}$ & $q\left(10^{-7} \mathrm{~m}^{3} \mathrm{~s}^{-1}\right)$ & $T(\mathrm{~K})$ \\
\hline 1 & 0,938 & 0,08 & 3,24 & 298 \\
2 & 0,951 & 0,36 & 3,16 & 298 \\
3 & 0,951 & 0,7 & 3,02 & 298 \\
4 & 0,939 & 0,17 & 3,23 & 308 \\
\hline
\end{tabular}

Em contrapartida, não há uma diferença apreciável no perfil de ruptura para o $\mathrm{CH}_{4}$, o que indica que os dados experimentais apresentados pelo autor de referência (Delgado et al., 2006) não são suficientes para uma validação rigorosa, uma vez que eles podem levar a modelos que não satisfaçam o balanço de massa.

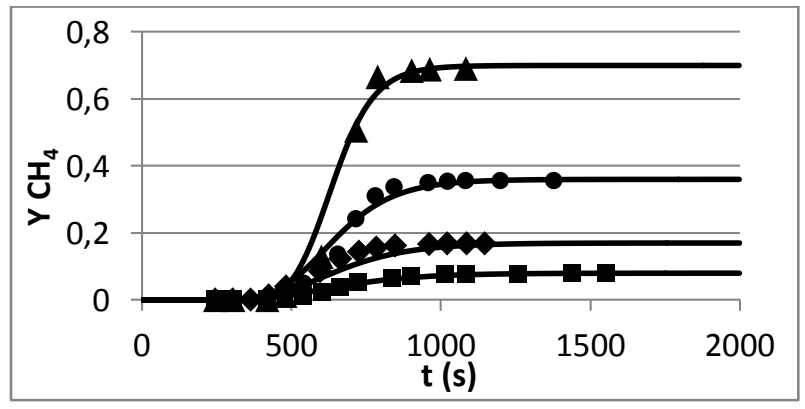

Figura 1 - Curvas de ruptura misturas $\mathrm{CH}_{4} / \mathrm{N}_{2}$ em silicalita para os casos de validação na Tabela 3 . Quadrados; Caso 1. Círculos; Caso 2. Triângulos; Caso 3. Losangos Caso 4.

Desta forma, seria importante uma comparação com dados experimentais de curva para N2 ou, alternativamente, comparar dados de adsorção e dessorção do $\mathrm{CH}_{4}$, conforme mostrado na Figura 2 (c). Neste sentido, os pontos fornecidos pelo autor em referência são úteis para uma verificação menos rígida da modelagem implementada, mas a validação do modelo carece de um trabalho 
experimental mais completo. Também foi analisado o efeito de competição por sítios ativos no leito de silicalita, durante o fenômeno de adsorção de misturas binárias de $\mathrm{N}_{2}-\mathrm{CH}_{4}$.

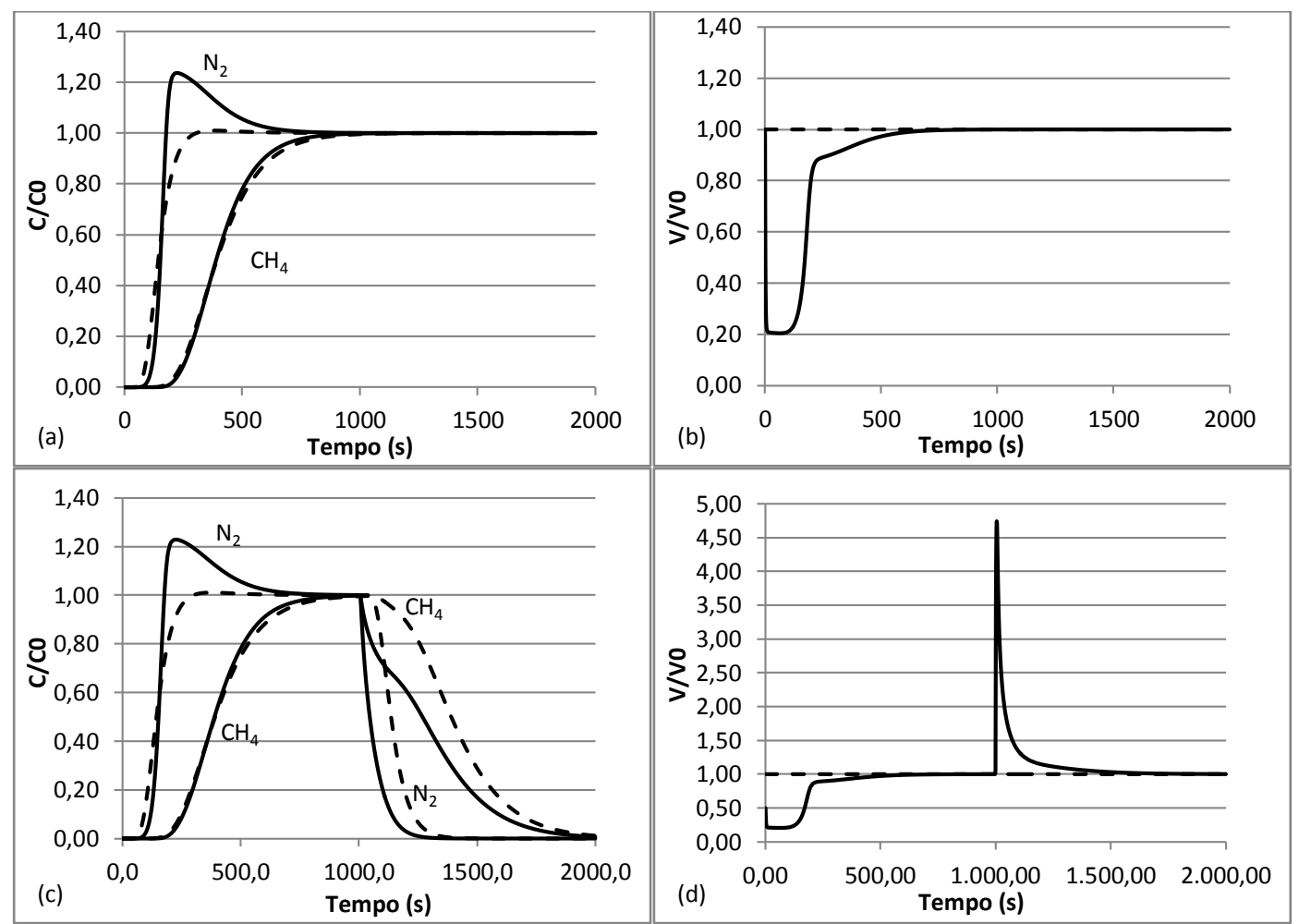

Figura 2 - Perfis de concentração e velocidade na saída do leito à velocidade variável (linha cheia) e velocidade constante (linha tracejada). Curvas puramente de adsorção;(a) e (b) e adsorção até $1000 \mathrm{~s}$ seguida de dessorção (c) e (d)

A Figura 3 apresenta os perfis de adsorção-dessorção para a fase fluida e sólida em um leito a 1,0 bar e $25^{\circ} \mathrm{C}$, alimentado por uma mistura contendo $20 \%$ em metano. Apesar de a literatura ter apresentado apenas dados de adsorção de componente puro para tal adsorvente, o efeito da adsorção de misturas foi modelado pela isoterma de Langmuir com competição com dados de substâncias puras. Como pode ser observado na Figura 3 (a), as diferenças observadas entre os perfis obtidos com e sem competição são muito pequenas, porém, na Figura 3 (b) foi possível observar que a quantidade adsorvida de metano foi maior utilizando a isoterma sem competição, demostrando que a desconsideração do efeito de competição pode levar a uma superestimação da quantidade adsorvida dos gases em estudo.

É sabido que os processos de adsorção são inerentemente exotérmicos, liberando energia na forma de calor à medida que a adsorção ocorre e, por outro lado, um aumento na temperatura desfavorece o equilíbrio no sentido da adsorção. Devido a este fato, foi realizada uma avaliação da influência da pressão total, em um leito com parede a $25^{\circ} \mathrm{C}$. Conforme demonstrado na Figura 4 , a variação da pressão do leito afeta fortemente o pico de temperatura na saída da coluna, com variações 
tão altas quanto $6^{\circ} \mathrm{C}$ em relação à entrada. Tal fato é uma consequência direta da quantidade adsorvida, que, na porção linear da isoterma é tão grande quanto maior a pressão for, o que demonstra a importância desta variável para o projeto de uma unidade experimental de adsorção. Também na Figura 4 são apresentados os perfis axiais de temperatura ao longo da etapa de adsorção nos tempos de 2, 35, 68, 102 e 135 segundos na pressão de 1,0 bar (c) e 3,0 bar (d). Nela é possível perceber que a magnitude da frente de calor se mantém constante ao longo do leito. No entanto, em condições que causem uma maior dissipação axial do calor, tal magnitude tende a diminuir com o avanço em $\mathrm{z}$. Portanto, é recomendável o posicionamento de um termopar no inicio do leito para captar a região de maior temperatura.
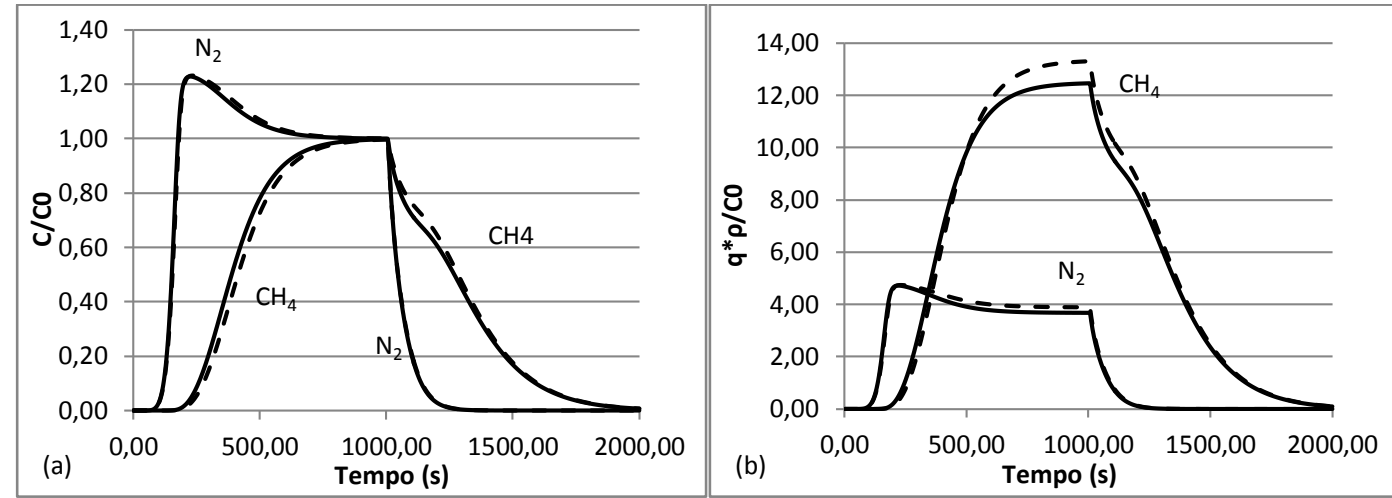

Figura 3 - Curvas de adsorção-dessorção de $\mathrm{CH}_{4}$ e $\mathrm{N}_{2}$ em fase gasosa (a) e adsorvida (b) utilizando a isoterma de Langmuir com competição (linha sólida) e sem competição (linha tracejada).

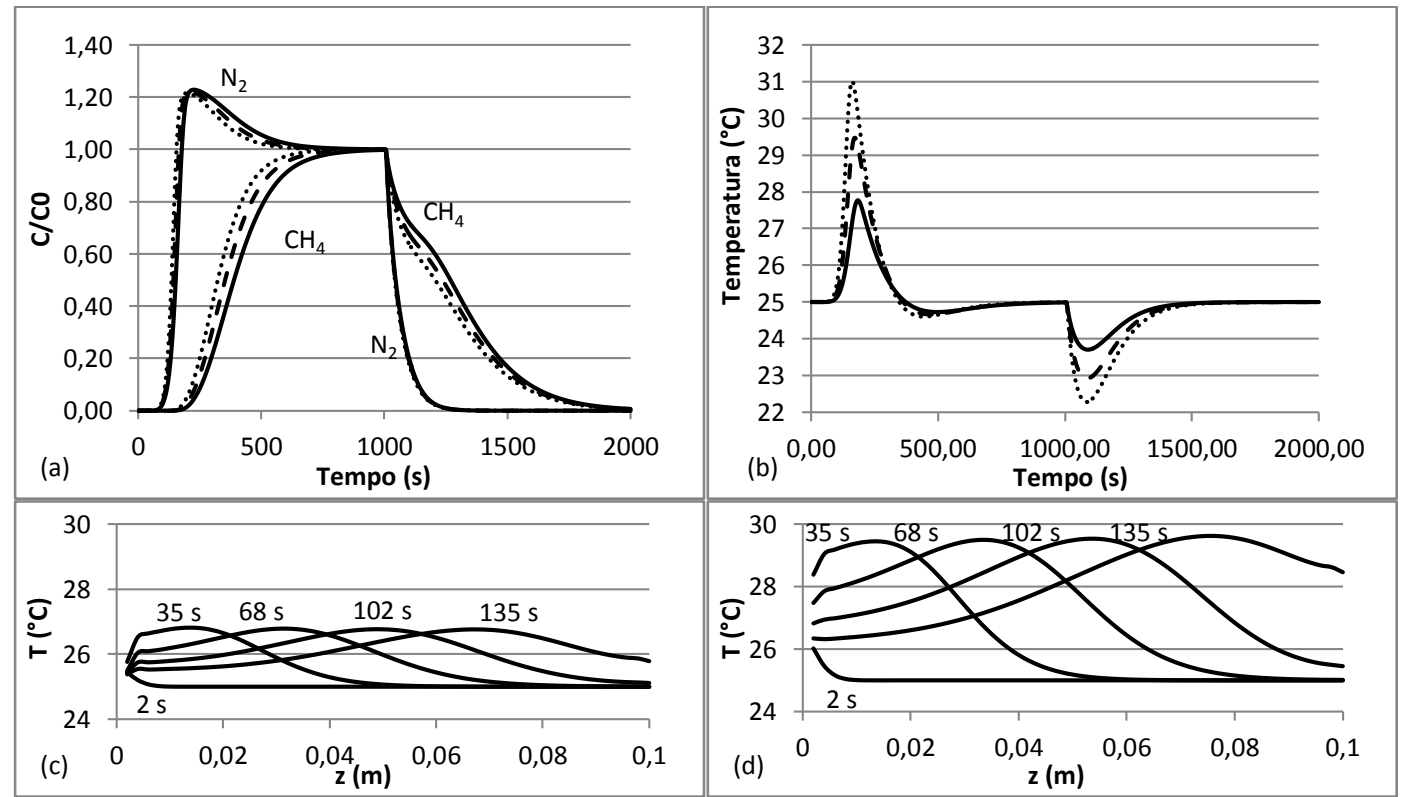

Figura 4 - Perfis de (a) composição e (b) temperatura a 1,0 bar (linha cheia), 2,0 bar (linha tracejada) e 3,0 bar (linha pontilhada). Temperaturas axiais em um leito a (c) 1,0 bar e (d) 3,0 bar. 


\section{9 a 22 de outubro de 2014 \\ Florianópolis/SC}

Provavelmente maiores diferenças sejam observadas para leitos com diâmetros em escala industrial. No entanto, para melhor representação do fenômeno em tais escalas, é fundamental considerar que o calor será gerado no interior das partículas e deverá ser propagado até a parede do leito. Desta forma, é importante aprimorar o modelo matemático de modo a contemplar a inércia térmica das partículas adsorventes.

\section{CONCLUSÃO}

A simulação da dinâmica de leitos fixos para a separação de gases efluentes de tratamento de gás natural é de suma importância para dimensionar corretamente a capacidade de equipamentos dessa natureza, evitando assim esforços experimentais desnecessários. Dentre as análises do Capítulo 3, foi observado que a inclusão do balanço de massa global no conjunto de equações é imprescindível para a simulação do comportamento da coluna de adsorção, quando esta está sujeita à alimentação de misturas de componentes não inertes. Os resultados obtidos neste trabalho estão sendo usados para nortear o projeto e montagem de um sistema experimental para investigação do comportamento de leitos de adsorção para a remoção de contaminantes do gás natural.

\section{BIBLIOGRAFIA}

DELGADO, J.A. UGUINA M.A. SOTELO, J.L. RUÍZ, B. Modelling of the fixed-bed adsorption of methane/nitrogen mixtures on silicalite pellets. Separation and Purification Technology, Vol 50, 2006, Pg. 192-203

DE WASCH, A.P. FROMENT, G.F. Heat transfer in packed beds, Chemical Engineering Science, vol. 27, 1972, pg. 567-576

JONES, J.K. STACEY, J.M. The recovery and liquefaction of helium from natural gas in Poland, Cryogenics Volume 14, Ed 4, Abril 1974, Pg. 198-202.

KIDNAY, A.J. PARRISH, W.R. Fundamentals of Natural Gas Processing. Florida: CRC, 2006. 418p.

KUO, J.C. WANG, K.H. CHEN, C. Pros and cons of different Nitrogen Removal Unit (NRU) technology, Journal of Natural Gas Science and Engineering, Volume 7, 2012, Pg 52-59

LOKHANDWALA, K.A. PINNAU, I. HE, Z. AMO, K.D. DACOSTA, A.R. WIJMANS, J.G.

BAKER, R.W. Membrane separation of nitrogen from natural gas: A case study from membrane synthesis to commercial deployment, Journal of Membrane Science, Volume 346, Ed. 2, 15 janeiro 2010, Pg. 270-279.

MADEIRA, A.C.F. Avaliação da tecnologia de adsorção "psa" para remoção de nitrogênio do gás natural, Tese de doutorado, EQ/UFRJ, Agosto de 2008, 116 p.

PETZOLD, L.R. DDASL. 15 de março de 1983, EUA/CA

RUTHVEN, D.M. Principles of adsorption and adsorption processes. EUA: Wiley-Interscience, 1984. $433 \mathrm{pg}$.

TAGLiABUE,M. FARRUSSENG, D. VALENCIA, S. AGUADO, S. RAVON, U. RIZZO, C. CORMA, A. MIRODATOS, C. Natural gas treating by selective adsorption: Material science and chemical engineering interplay, Chemical Engineering Journal, Volume 155, Ed. 3, 15 dezembro 2009, Pg. 553-566. 